\title{
Targeting of Endothelin Receptors in the Healthy and Infarcted Rat Heart Using the PET Tracer ${ }^{18}$ F-FBzBMS
}

\author{
Takahiro Higuchi ${ }^{1}$, Christoph Rischpler ${ }^{1}$, Kenji Fukushima ${ }^{1}$, Takuro Isoda ${ }^{1}$, Jinsong Xia $^{1}$, Mehrbod S. Javadi ${ }^{1}$, \\ Zsolt Szabo ${ }^{1}$, Robert F. Dannals ${ }^{1}$, William B. Mathews ${ }^{1}$, and Frank M. Bengel ${ }^{1,2}$ \\ ${ }^{I}$ Division of Nuclear Medicine, Russell H. Morgan Department of Radiology, Johns Hopkins University, Baltimore, Maryland; and \\ ${ }^{2}$ Department of Nuclear Medicine, Hannover Medical School, Hannover, Germany
}

The endothelin subtype-A receptor (ET-A) is a promising therapeutic target in cardiovascular disease. We sought to determine the feasibility of an ${ }^{18} \mathrm{~F}$-labeled ligand, ${ }^{18} \mathrm{~F}-(\mathrm{N}-[[29-$ [[(4,5-dimethyl-3-isoxazolyl)amino]sulfonyl]-4-(2-oxazolyl)[1,19biphenyl]-2-yl]methyl]- $N, 4$-fluorobenzamide) ( $\left.{ }^{18} \mathrm{~F}-\mathrm{FBzBMS}\right)$, for imaging ET-A in the healthy and injured rat heart. Methods: Male Wistar rats were used for all experiments. The specificity of cardiac ${ }^{18} \mathrm{~F}-\mathrm{FBzBMS}$ uptake was determined in healthy animals $(n=23)$ using pretreatment with various blocking agents and doses. Myocardial infarction (MI) was induced by permanent left coronary ligation in 32 animals. Autoradiography was conducted to determine regional FBzBMS distribution relative to tissue perfusion at various times after MI. Histology and immunohistochemistry were performed for validation. The feasibility of in vivo detection of the tracer signal was tested using dedicated small-animal PET $(n=6)$. Results: At autoradiography, intravenous pretreatment with the selective ET-A blocker BMS-207940 reduced myocardial FBzBMS uptake by $93 \% \pm$ $0.7 \%$. Oral pretreatment with the clinical blocker bosentan resulted in a dose-dependent partial blockade $(5 \mathrm{mg} / \mathrm{kg}, 48 \% \pm$ $6 \% ; 50 \mathrm{mg} / \mathrm{kg}, 61 \% \pm 7 \%$; and $100 \mathrm{mg} / \mathrm{kg}, 88 \% \pm 0.7 \%)$. After MI, FBzBMS uptake was preserved in the infarct region from day 1 to month 6 , whereas the perfusion tracer ${ }^{201} \mathrm{Tl}$ showed a persistent defect (Ml-to-remote ratios: ${ }^{201} \mathrm{TI}, 0.23 \pm$ $0.28,0.39 \pm 0.07,0.31 \pm 0.07,0.24 \pm 0.12,0.29 \pm 0.10$, and $0.23 \pm 0.09$; and FBzBMS, $0.94 \pm 0.28,0.92 \pm 0.20,0.88 \pm$ $0.13,0.82 \pm 0.12,0.80 \pm 0.11$, and $0.84 \pm 0.08$ at day 1 , day 3 , week 1 , month 1 , month 2 , and month 6 , respectively) $(P<0.01$ vs. $\left.{ }^{201} \mathrm{TI}\right)$. Ex vivo analysis confirmed ET-A expression in the infarct area, where the signal was partially colocalized with CD31 expression on endothelial cells. In vivo small-animal PET successfully confirmed specific uptake and blockade of FBzBMS in healthy myocardium. Conclusion: Cardiac uptake of the PET tracer ${ }^{18} \mathrm{~F}-\mathrm{FBzBMS}$ is specific for ET-A expression in rats, shows infarct-related alterations, and can be imaged noninvasively. Further efforts to establish myocardial ET-A imaging methodology are warranted, with the perspective of determining role, efficacy, and benefit of ET-A targeted drug treatment in cardiovascular disease.

\footnotetext{
Received Mar. 16, 2012; revision accepted Sep. 17, 2012.

For correspondence contact: Frank M. Bengel, Department of Nuclear Medicine, Hannover Medical School, Carl-Neuberg-Strasse 1, D-30625 Hannover, Germany.

E-mail: bengel.frank@mh-hannover.de

Published online Jan. 11, 2013

COPYRIGHT @ 2013 by the Society of Nuclear Medicine and Molecular Imaging, Inc.
}

Key Words: myocardial infarction; endothelin receptor; molecular imaging; PET

J Nucl Med 2013; 54:277-282

DOI: 10.2967/jnumed.112.106096

\section{$\mathbf{E}$} ndothelins are potent vasoconstrictors that are released by endothelial cells (1). Endothelin-1 is the predominant isoform relevant to the cardiovascular system, and stimulation of the endothelin subtype-A receptor (ET-A) mediated by endothelin-1 has effects beyond vascular tissue. These effects include positive myocardial inotropy, growth promotion of cardiac fibroblasts, acceleration of cardiac hypertrophy, and arrhythmogenesis in various pathologic conditions $(2,3)$. In experimental heart failure models, nonselective ET-A and endothelin subtype-B receptor (ET-B) antagonism and selective ET-A antagonism exerted beneficial effects on left ventricular remodeling $(4,5)$. In humans with end-stage heart failure, cardiac tissue endothelin-1 levels and ET-A levels are increased (6). Because recognition of the importance of the endothelin system in heart failure is expanding, several clinical multicenter trials with ET-A antagonist have been initiated. Results of those trials, however, have been inconclusive and highlight the need for further optimization of the therapeutic strategy with regards to timing, dosage, and patient selection $(2,7)$.

Noninvasive imaging of cardiac ET-A expression may be a promising means not only to understanding the dynamic process of ventricular remodeling after myocardial infarction but also to optimizing ET-A antagonist therapy. Recently, synthesis and initial in vivo application of the ${ }^{18} \mathrm{~F}$-labeled ET-A antagonist ${ }^{18} \mathrm{~F}-(N$-[[29-[[(4,5-dimethyl-3-isoxazolyl)amino] sulfonyl]-4-(2-oxazolyl)[1,19-biphenyl]-2-yl]methyl]- $N$,4fluorobenzamide) ( $\left.{ }^{18} \mathrm{~F}-\mathrm{FBzBMS}\right)$ have been reported $(8)$. The aim of this study was to determine the feasibility of using this molecular imaging agent to study cardiac ET-A expression in rats.

\section{MATERIALS AND METHODS}

\section{Animals}

The protocols were approved by the Johns Hopkins Animal Care and Use Committee and conformed to the Guide for the Care and Use of Laboratory Animals (9). 
Sixty-one male Wistar rats, weighing 250-350 g, were used for experiments. The rats were anesthetized using 1\%-3\% isoflurane and mechanical ventilation.

In a subgroup of 32 animals, myocardial infarction was induced using permanent coronary ligation. Left thoracotomy was performed to expose the heart. A 7-0 polypropylene suture was passed through the myocardium beneath the proximal portion of the left coronary artery, and the snare was ligated to occlude the left coronary artery (10). The success of coronary occlusion was confirmed by regional cyanosis of the myocardial surface. Then, the chest was closed, and the animals were allowed to recover. The rats were given intramuscular buprenorphine $(0.5 \mathrm{mg} / \mathrm{kg})$ twice within $24 \mathrm{~h}$ for postoperative analgesia.

\section{Biodistribution and Autoradiography}

${ }^{18} \mathrm{~F}-\mathrm{FBzBMS}$ was synthesized as previously described (8). The following 6 groups of healthy rats were studied: group 1, controls without pretreatment before tracer injection $(n=5)$; group 2, intravenous pretreatment with the specific ET-A antagonist BMS$207940(2 \mathrm{mg} / \mathrm{kg})$, for complete blockage of ET-A $(n=4)$; groups 3,4 , and 5, oral pretreatment with the nonspecific, clinically approved ET-A and ET-B antagonist bosentan at doses of 5, 50, and $100 \mathrm{mg} / \mathrm{kg}$ ( $n=3$ per dose), respectively; and group 6 , oral pretreatment with the angiotensin receptor antagonist valsartan $(50 \mathrm{mg} / \mathrm{kg})(n=5)$. Fifteen minutes after the pretreatment, ${ }^{18} \mathrm{~F}-\mathrm{FBzBMS}$ (37 MBq) was injected via the tail vein. Ninety minutes after tracer injection, the rats were euthanized. Blood was collected, and organs (heart, lung, liver, kidney, skeletal muscle) were quickly removed for $\gamma$-counting (Wizard automatic $\gamma$-counter; Perkin Elmer). Hearts were then subjected to autoradiography as described below.

Dual-tracer autoradiography was performed for simultaneous assessment of regional myocardial perfusion with ${ }^{201} \mathrm{Tl}$ chloride and ${ }^{18} \mathrm{~F}$-FBzBMS uptake $(11,12)$. The rat model of permanent coronary occlusion was studied using groups of animals after 1 $\mathrm{d}(n=5), 3 \mathrm{~d}(n=5), 1 \mathrm{wk}(n=7), 1 \mathrm{mo}(n=6), 2 \mathrm{mo}(n=4$, and 6 mo $(n=4) .{ }^{18}$ F-FBzBMS $(111 \mathrm{MBq})$ was injected via the tail vein. After an uptake phase of $90 \mathrm{~min}$, a small dose of ${ }^{201} \mathrm{Tl}$ chloride (0.74 MBq) was injected. Animals were then sacrificed, and the heart was removed, rinsed, frozen, and sliced into $20-\mu \mathrm{m}$ short-axis sections, which were used for autoradiography and histologic analysis. Using a digital autoradiography system (Cyclone Plus Phosphor Imager; Perkin Elmer), we exposed the first slice within $1-2 \mathrm{~h}$ of sacrifice for $30 \mathrm{~min}$, for visualization of ${ }^{18} \mathrm{~F}$ FBzBMS distribution. After $2 \mathrm{~d}$ to allow for complete decay of ${ }^{18} \mathrm{~F}$, a second slice was exposed for $2 \mathrm{wk}$, for visualization of ${ }^{201} \mathrm{Tl}$ distribution (13). Region-of-interest analysis of digital images was performed to determine activity ratios for the remote area relative to the infarct area.

For validation, histologic tissue analysis was performed. Shortaxis slices were stained with hematoxylin and eosin to determine scar localization and extent. Additionally, immunohistochemical staining and immunofluorescence microscopy were performed in rat hearts $1 \mathrm{wk}$ after infarction, using standard techniques. Sheep polyclonal anti-ET-A antibody (Abcam) and mouse monoclonal anti-rat CD31 antibody (Milipore) were used as primary antibodies for staining.

\section{In Vivo PET}

A dedicated small-animal PET scanner (eXplore VISTA; GE Healthcare) and small-animal CT scanner (X-SPECT/CT; Gamma Medica) were used. Healthy rats were imaged without pretreat- ment $(n=3)$ and after oral pretreatment with bosentan $(100 \mathrm{mg} / \mathrm{kg}$; $n=3$ ). Static PET scans were acquired for $30 \mathrm{~min}$, after an uptake phase of $1 \mathrm{~h}$ after injection of $37 \mathrm{MBq}$ of ${ }^{18} \mathrm{~F}-\mathrm{FBzBMS}$. A CT scan was acquired immediately after the PET scan as a reference for localization of the heart.

\section{Statistical Analysis}

All results are expressed as mean \pm SD. Statistical analysis was performed with StatMate III (ATMS Co., Ltd.). Multiple-group comparisons were made using the Kruskal-Wallis test, followed by the Dunn multiple-contrast hypothesis test to identify differences between each set of groups. A $P$ value of less than 0.05 was considered statistically significant.

\section{RESULTS}

\section{${ }^{18}$ F-FBzBMS Specificity and Effects of Medication}

At autoradiography, ${ }^{18} \mathrm{~F}-\mathrm{FBzBMS}$ uptake in healthy rats was homogeneous throughout the left ventricular wall. Biodistribution studies showed that radiotracer activity at $90 \mathrm{~min}$ after tracer injection was $0.039 \% \pm 005 \%, 0.014 \% \pm 001 \%$, $0.043 \% \pm 012 \%, 0.085 \% \pm 013 \%, 0.992 \% \pm 302 \%$, and $0.008 \% \pm 0.001 \%$ injected dose per gram for heart, blood, lung, kidney, liver, and skeletal muscle, respectively.

After intravenous pretreatment with the specific ET-A antagonist BMS-207940, organ uptake was $0.018 \% \pm 004 \%$, $0.104 \% \pm 028 \%, 0.029 \% \pm 009 \%, 0.045 \% \pm 010 \%$, $0.977 \% \pm 169 \%$, and $0.012 \% \pm 0.003 \%$ injected dose per gram for heart, blood, lung, kidney, liver, and skeletal muscle, respectively. A significant decrease, compared with the unblocked state, was observed for the heart $(P<0.01)$ and kidneys $(P<0.05)$, whereas a significant increase occurred in the blood $(P<0.001)$. Overall, the ratio of heart to blood radioactivity was reduced by $93 \% \pm 1 \%$, confirming the high specificity of cardiac uptake (Fig. 1A and 1B).

Additionally, oral medication with bosentan reduced the ratio of heart to blood ${ }^{18} \mathrm{~F}-\mathrm{FBzBMS}$ uptake in a dosedependent manner $(5 \mathrm{mg} / \mathrm{kg}, 48 \% \pm 6 \% ; 50 \mathrm{mg} / \mathrm{kg}, 61 \% \pm$ $7 \%$; and $100 \mathrm{mg} / \mathrm{kg}, 88 \% \pm 0.7 \%$ ), whereas there was no reduction by pretreatment with oral valsartan as an angiotensin receptor blocker, which does not have direct effects on the ET (Fig. 1B).

\section{Regional ${ }^{18}$ F-FBzBMS Distribution After Myocardial Infarction}

${ }^{201} \mathrm{Tl}$ autoradiography of myocardial perfusion showed a regional defect, which corresponded to the histologically defined infarct area (Fig. 2). On the other hand, ${ }^{18} \mathrm{~F}$ FBzBMS uptake was clearly present within the ${ }^{201} \mathrm{Tl}$ defect. This uptake was observed in the infarct area at all time points, from day 1 to month 6 . The remote-to-infarct area ratio was significantly higher for ${ }^{18} \mathrm{~F}-\mathrm{FBzBMS}$ than for ${ }^{201} \mathrm{Tl}\left({ }^{201} \mathrm{Tl}: 0.23 \pm 0.28,0.39 \pm 0.07,0.31 \pm 0.07,0.24 \pm\right.$ $0.12,0.29 \pm 0.10$, and $0.23 \pm 0.09$, at day 1 , day 3 , week 1 , month 1 , month 2 , and month 6 , respectively; and ${ }^{18} \mathrm{~F}$ FBzBMS: $0.94 \pm 0.28,0.92 \pm 0.20,0.88 \pm 0.13,0.82 \pm$ $0.12,0.80 \pm 0.11$, and $0.84 \pm 0.08$ at day 1 , day 3 , week 1 , month 1 , month 2 , and month 6 , respectively) $(P<0.01$ ${ }^{18}$ F-FBzBMS vs. ${ }^{201} \mathrm{Tl}$ ). 


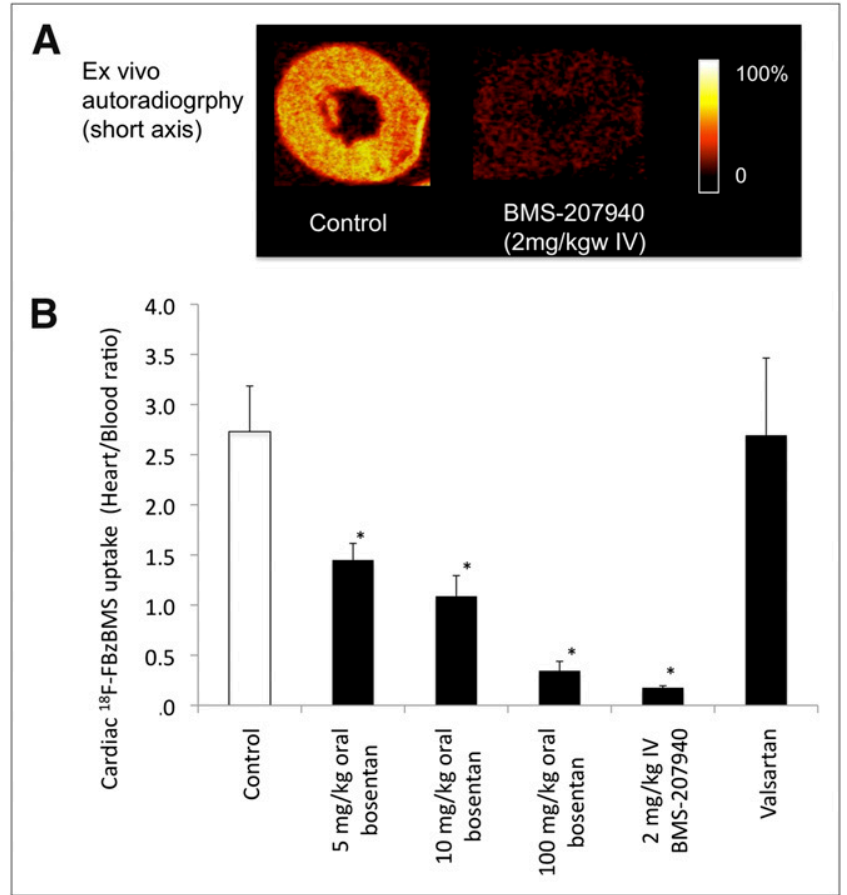

FIGURE 1. (A) Autoradiographic short-axis images of ${ }^{18} \mathrm{~F}$ FBzBMS in healthy rat hearts with and without ET-A antagonist BMS-207940 pretreatment. (B) Effects of medication on cardiac ${ }^{18}$ F-FBzBMS uptake. ${ }^{\star} P<0.001$ vs. control. $\mathrm{mg} / \mathrm{kgw}=\mathrm{mg}$ per $\mathrm{kg}$ of body weight.

ET-A expression in the infarct area was confirmed by immunohistochemical staining (Fig. 3). Although the ET-A signal in nondamaged remote myocardium comes mostly from diffuse, mild ET-A expression on myocytes and other cells, multiple labeling colocalized a large fraction of the ET-A signal within the infarct area (where viable myocytes are mostly absent) to vascular cells expressing the endothelial marker CD31.

\section{In Vivo PET}

${ }^{18} \mathrm{~F}-\mathrm{FBzBMS}$ uptake was robustly detected in healthy hearts in vivo, using small-animal PET and CT (Fig. 4). Consistent with autoradiography, cardiac uptake was abolished by pretreatment with oral bosentan, confirming the specificity of ${ }^{18} \mathrm{~F}-\mathrm{FBzBMS}$ for visualization of ET receptor expression.

\section{DISCUSSION}

This study demonstrates the feasibility of measuring cardiac ET-A expression using the PET tracer ${ }^{18} \mathrm{~F}-$ FBzBMS. Homogeneous distribution of the tracer signal was observed in healthy myocardium, and pretreatment with ET-A antagonists specifically reduced tracer uptake. After permanent coronary occlusion, tracer uptake in the hypoperfused infarct area was preserved and validated by histologic proof of ET-A expression.

Like the adrenergic and renin-angiotensin-aldosterone systems, the endothelin system is amenable to clinical phar- macologic blockade and seems to play an important role in the development and progression of cardiovascular disease. It therefore also constitutes an attractive target for molecular imaging. Upregulation of tissue and plasma endothelin levels has been reported in heart failure patients $(6,14)$. Experimental work first confirmed that pharmacologic blockade can be beneficial. Shimoyama et al. used the clinically approved nonselective ET-A and ET-B antagonist bosentan in a dog model of chronic heart failure and found improved overall left ventricular function associated with reduced systemic vascular resistance after short-term treatment (15). Long-term treatment with either selective ET-A or nonselective ET-A and ET-B antagonists also prevented left ventricular remodeling in rat heart failure models $(4,5,16,17)$. Mulder et al. showed an incremental effect of long-term ETA antagonist treatment on top of angiotensin-converting enzyme inhibitor medication, for improving hemodynamics and survival in a rat model of myocardial infarction (18). Although these experimental studies suggested a beneficial effect, long-term endothelin system blockade has not yet been conclusively established in the clinical setting, despite several clinical trials (2). The Research on Endothelin Antagonism in Chronic Heart Failure (REACH-1) trial demonstrated a reduction of death and an improvement of heart failure with bosentan (500 mg twice daily), although an elevation of liver enzymes was observed (19). A subsequent trial with smaller doses to prevent liver damage failed to

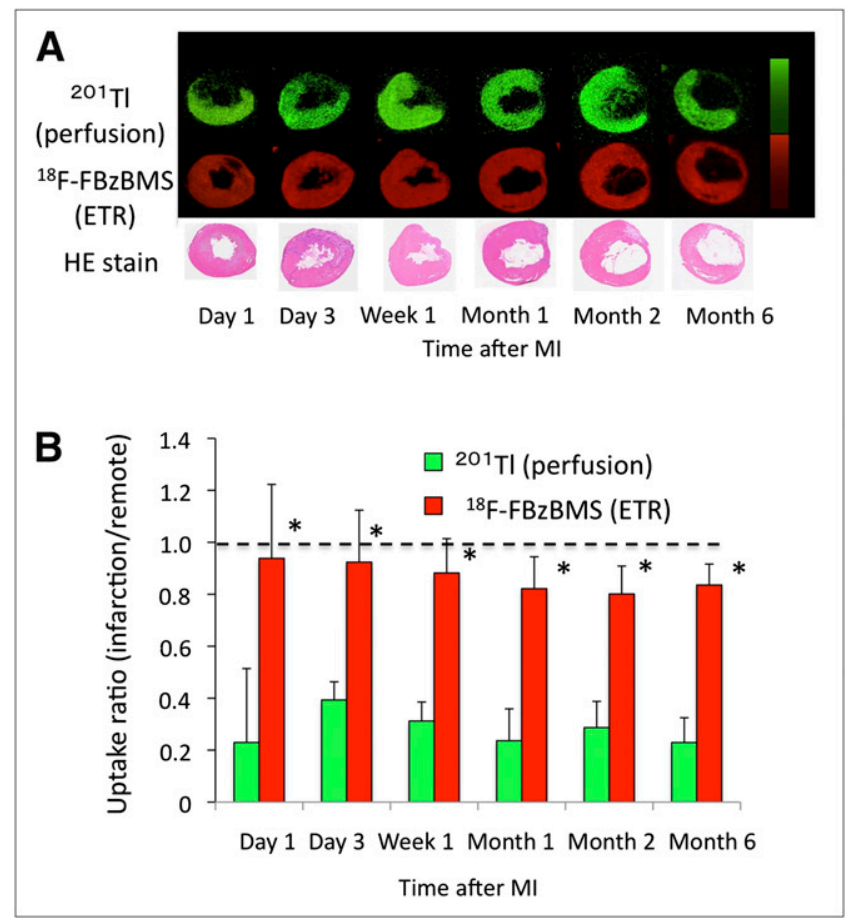

FIGURE 2. (A) Representative autoradiographic short-axis images of ${ }^{18} \mathrm{~F}-\mathrm{FBzBMS}$ at different time points after permanent left coronary occlusion. (B) Time course of ratio of infarct myocardial uptake to remote myocardial uptake for ${ }^{18} \mathrm{~F}-\mathrm{FBzBMS}$ after permanent left coronary occlusion. ${ }^{18} \mathrm{~F}-\mathrm{FBzBMS}$ uptake was preserved in infarct region from day 1 to month 6 , whereas perfusion tracer ${ }^{201} \mathrm{TI}$ showed persistent defect. ${ }^{*} P<0.001$ vs ${ }^{201} \mathrm{Tl}$. 


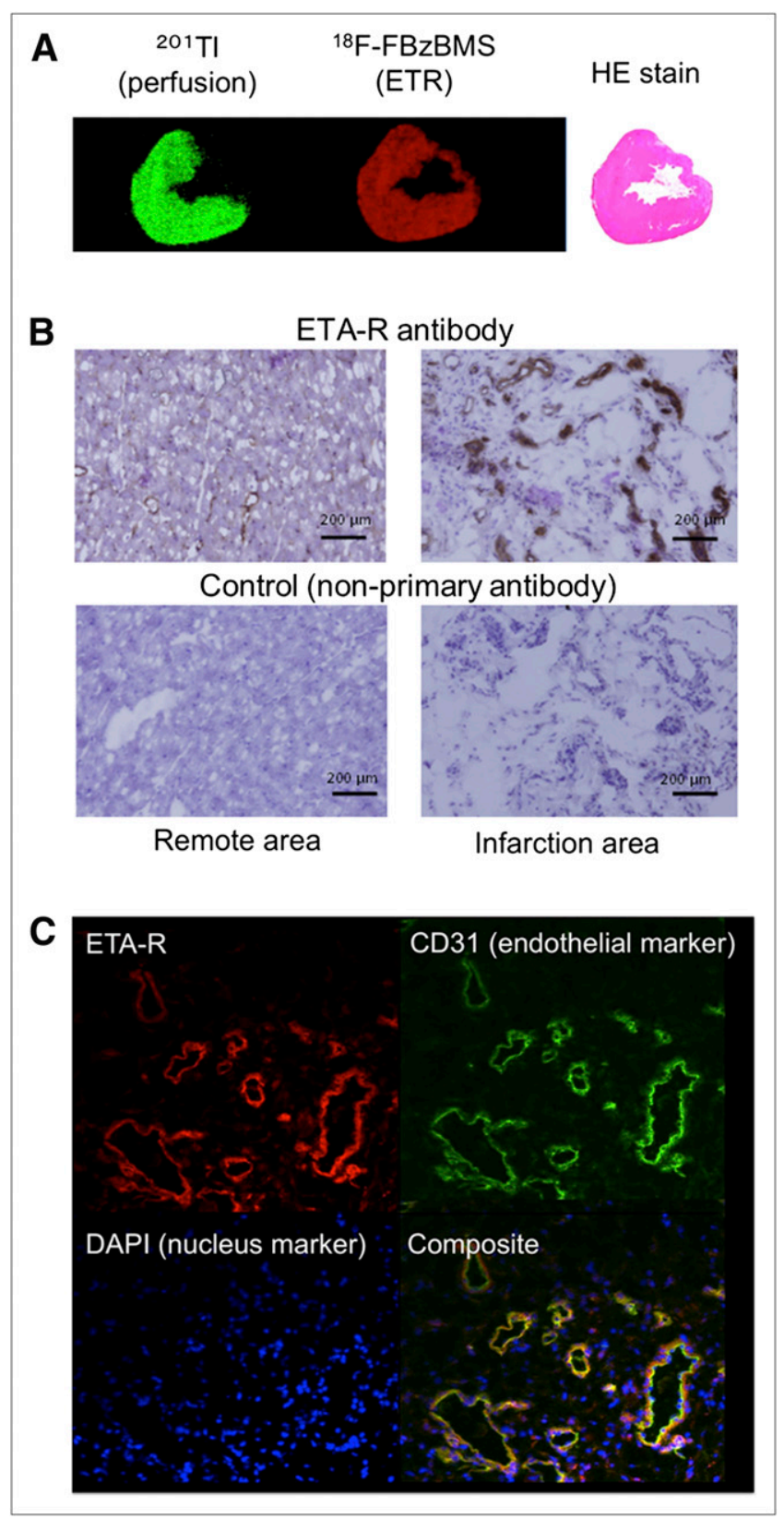

FIGURE 3. (A) Autoradiography shows regional uptake of ET-A targeted tracer ${ }^{18} \mathrm{~F}-\mathrm{FBzBMS}$ (red, middle) in infarct region (characterized by thin wall on histologic hematoxylin and eosin stain), which is comparable to remote myocardium and more pronounced than regional perfusion tracer uptake (green, left). (B) Immunohistochemistry of representative sections from remote (left) and infarct region (right) show diffuse mild brown stain of viable myocytes and vessels in remote region, whereas there is strong signal from vessels and absent signal from vacuolar nonviable tissue in infarct region. Control images without primary antibody confirm absence of specific signal (brown) in both regions. (C) Multiple labeling immunofluorescence imaging of representative section from infarct region demonstrates colocalization of ET-A with endothelial marker CD-31 in large fraction of cells. Presence of ET-A signal in some areas that are not matched to CD-31 indicates existence of small fraction of additional ET-A-positive (probably inflammatory) cells. demonstrate similar beneficial effects (20). More recently, the EndothelinA Receptor Antagonist Trial in Heart Failure demonstrated no significant improvement of left ventricular function or mortality in any dose using the selective ET-A antagonist darusentan in chronic heart failure patients (7). These results emphasize the need for an improved understanding of the role of endothelin system activation in patients with heart failure. They may also serve as a background for potential future applications of molecular imaging to monitor the efficacy of blockade and thereby optimize the therapeutic dose individually while minimizing the risk of side effects. A recent experimental study, for example, showed that, when selective ET-A antagonist was started in the early phase within $3 \mathrm{~h}$ of myocardial infarction in rats, a deleterious effect was observed on left ventricular remodeling. Infarct expansion occurred via reduced collagen deposit and matrix metalloproteinase activation, highlighting the significance of timing of antagonist treatment (21).

The present study supports the feasibility of ${ }^{18} \mathrm{~F}-$ FBzBMS for imaging ET-A in the rat heart by use of autoradiography, biodistribution studies, and in vivo imaging in the unblocked and blocked states. The results are in line with a prior publication by Mathews et al. (8), in which biodistribution in mice and in vivo kinetics in a monkey were reported. Our study expands on the prior work by focusing on the heart as the imaging target and by analyzing the effect of myocardial infarction on ${ }^{18} \mathrm{~F}$ FBzBMS uptake. For this purpose, we chose a model of permanent left coronary occlusion. This model results in death of myocytes and development of transmural scarring in the area supplied by the occluded artery. Accordingly, most of the ET-A signal in the infarct area comes from nonmyocyte cells and colocalizes with endothelial cells, suggesting that ET-A may play a role in vessel formation as part of scar development. Some of the signal also localized to nonendothelial cells, suggesting a contribution of other cell types to the signal. This signal probably comes from inflammatory cells or fibroblasts, which can be found in the infarct region and which are known to express ET-A, albeit at a lower level than does the neovasculature $(22,23)$.

The latter observation also highlights a limitation of molecular myocardial imaging, namely that the tissue signal is not cell-specific but represents a mixture of signals coming from all cell types that are present in the respective region. The ET-A signal from the infarct area was similar in strength when compared with noninfarcted remote myocardium, with ratios being close to 1 . Yet, at immunohistochemistry, we observed a strong ET-A signal coming from vessels in the infarct region, which seems to be contradictory to the lack of regional autoradiographic signal increase. This discrepancy is best explained by the absence of viable myocytes in the infarct region. Myocytes express ET-A at a basic level $(3,24)$ and constitute most of the imaging signal in the remote and healthy myocardium. In the infarct region, however, myocytes are mostly absent, 


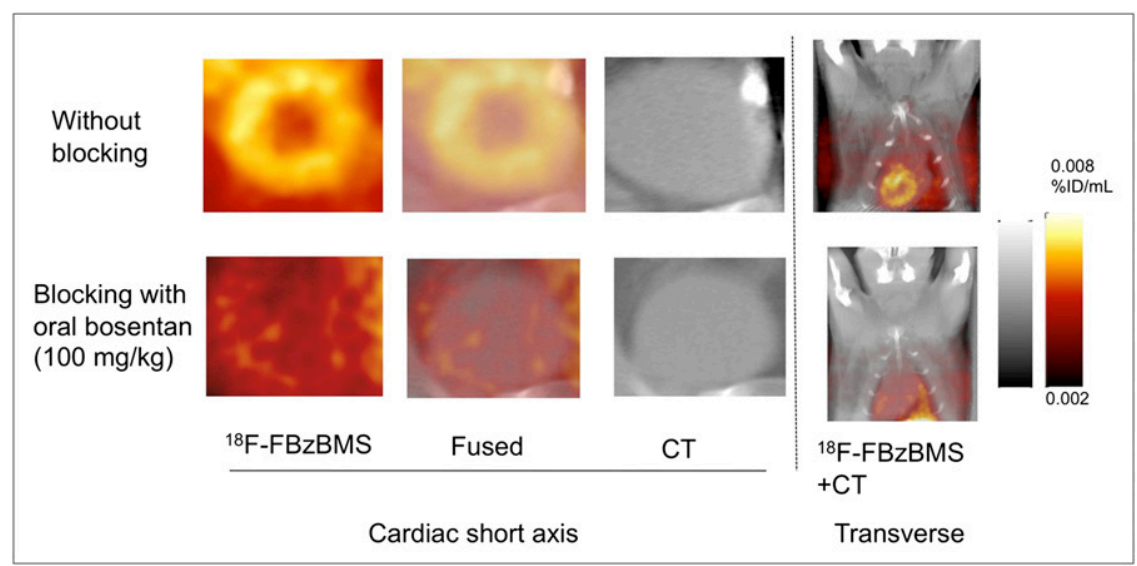

FIGURE 4. In vivo ${ }^{18} \mathrm{~F}-\mathrm{FBzBMS}$ PET and CT images in healthy rats. Images without blocking show regionally homogeneous myocardial uptake, without adverse effects by adjacent organs such as lungs and liver. Images after pretreatment with oral bosentan effectively abolish cardiac ${ }^{18} \mathrm{~F}-\mathrm{FBzBMS}$ uptake. $\%$ ID $=$ percentage injected dose. and all signal comes from the smaller number of activated endothelial cells, which show strong ET-A expression. The average ET-A imaging signal in the infarct tissue was thus comparable to remote myocardium.

However, the mild ET-A imaging signal from the infarct region is not consistent with the intense upregulation that has been reported for other biologic markers (13). The mild elevation of ${ }^{18} \mathrm{~F}-\mathrm{FBzBMS}$ signal above perfusion in the infarct area also persisted over time and did not show a peak in the early phase after infarction-a finding that is again different from the results of prior work using other tracers (13). This finding suggests that ET-A may not play a dominant role in the early healing phase after infarction and that its overall role may be limited after permanent occlusion.

More work, including other models of ischemic and nonischemic myocardial damage, is necessary to elucidate the contribution of ET-A to myocardial biology. Our preliminary results should be seen as a stimulus to implement molecular imaging in such studies. Heart-specific ET-A knockout mice have been developed recently $(24,25)$ and may offer a good model for further detailed assessment of ${ }^{18} \mathrm{~F}-\mathrm{FBzBMS}$ and its usefulness to study disease mechanisms. In those animals, in which only cardiomyocytes are deficient of ET-A, further insights into the endothelial cellspecific signal in the infarct area may be obtained, but such work clearly exceeds the frame of our present study.

Several tracers have previously been introduced for PET of endothelin receptors. ${ }^{11} \mathrm{C}-\mathrm{L}-753,037$, for example, is a nonselective tracer of ET-A and ET-B (26). It has been used to successfully visualize myocardium in mice and dogs. ET-A-selective blockade led to a significant reduction of tracer accumulation in the heart but not in the lungs, suggesting that cardiac uptake is predominately mediated by ET-A (27). An ${ }^{18}$ F-labeled nonselective tracer for ET-A and ET-B was introduced by Johnström et al. (28), and the same group later described a specific tracer for ET-B, ${ }^{18} \mathrm{~F}$ BQ3020, which showed binding in the lung and kidneys but not in the heart (29). Our agent, ${ }^{18} \mathrm{~F}-\mathrm{FBzBMS}$, has advantages for imaging of the heart in that it combines the longer half-life of ${ }^{18} \mathrm{~F}$ with a selectivity for ET-A as the relevant endothelin receptor in cardiac tissue.
Some limitations of the present study should be emphasized: our study focused on a reliable definition of signal strength, receptor specificity, and regional distribution of ${ }^{18} \mathrm{~F}$-FBzBMS in myocardium. First, most of the results were thus obtained using autoradiography and other ex vivo techniques. In vivo PET was performed in only a small subset of animals for proof of principle. Clearly, additional studies with an in vivo PET assay will be necessary to optimize imaging protocols, to clarify the feasibility of analysis of dynamic tracer kinetics, and to determine the in vivo course of ET-A expression after myocardial infarction. Furthermore, the influence of uptake on surrounding organs, including high liver and lung uptake, needs to be determined in more detail in subsequent studies. High liver uptake may, for example, interfere with imaging of regional myocardial tissue in the inferior wall because of scatter or reconstruction artifacts. Our study and prior work by Mathews et al. (8) give some support to the usefulness of regional myocardial imaging because they show regionally homogeneous uptake in healthy conditions, but feasibility for infarct imaging has not yet been proven. Second, the exact cell types responsible for the tracer uptake have not yet been identified. Although relatively strong ET-A expression on neovasculature is found in the infarct area at immunohistologic characterization, further experiments using micoautoradiography or fluorescent analogs of ${ }^{18} \mathrm{~F}$ FBzBMS need to be considered. Third, we did not perform in vivo imaging in our infarct model because of expected complications due to partial-volume effects resulting from wall thinning after permanent occlusion. We also did not implement more sophisticated approaches for modeling of dynamic imaging data. We believe that the development of such approaches is best achieved in larger animals, for which prior work with ${ }^{18} \mathrm{~F}$-FBzBMS has already suggested that kinetics can be accurately determined (8). Last, potential interspecies differences and issues related to permanent coronary occlusion need to be considered for translation to other animal models. But the present study should be seen as a first step toward a broader implementation of ET-A imaging in preclinical cardiac imaging studies. 


\section{CONCLUSION}

This proof-of-concept study supports the feasibility of the specific ET-A antagonist ${ }^{18} \mathrm{~F}$-FBzBMS for imaging of endothelin receptors in the rat heart. Results provide a rationale for subsequent studies to evaluate the potential of ET-A-targeted molecular imaging for studying the dynamic repair process after myocardial damage, for monitoring the efficacy of endothelin blockade, and for optimizing medication dose and timing individually.

\section{DISCLOSURE}

The costs of publication of this article were defrayed in part by the payment of page charges. Therefore, and solely to indicate this fact, this article is hereby marked "advertisement" in accordance with 18 USC section 1734 . The precursor for ${ }^{18} \mathrm{~F}-\mathrm{FBzBMS}$ was kindly provided by Dr. Natesan Murugesan under a material transfer agreement between the Johns Hopkins University and Bristol Myers Squibb Company. This project was supported in part by a 2008 Nuclear Cardiology Foundation Research Award and an American Heart Association Mid-Atlantic Affiliate Postdoctoral Fellowship Award. The work was also supported in part by NIH grants 1RO1HL092985, 5RO1DK050183, and 5U24CA092871-10. No other potential conflict of interest relevant to this article was reported.

\section{ACKNOWLEDGMENTS}

We are grateful to Jennifer Merrill, Roselle Abraham, Michael Bonios, James Fox, and Gilbert Green for their excellent research assistance.

\section{REFERENCES}

1. Barton M, Yanagisawa M. Endothelin: 20 years from discovery to therapy. Can J Physiol Pharmacol. 2008;86:485-498.

2. Ertl G, Bauersachs J. Endothelin receptor antagonists in heart failure: current status and future directions. Drugs. 2004;64:1029-1040.

3. MacCarthy PA, Grocott-Mason R, Prendergast BD, Shah AM. Contrasting inotropic effects of endogenous endothelin in the normal and failing human heart: studies with an intracoronary ET(A) receptor antagonist. Circulation. 2000;101:142-147.

4. Fraccarollo D, Hu K, Galuppo P, Gaudron P, Ertl G. Chronic endothelin receptor blockade attenuates progressive ventricular dilation and improves cardiac function in rats with myocardial infarction: possible involvement of myocardial endothelin system in ventricular remodeling. Circulation. 1997;96:3963-3973.

5. Rothermund L, Vetter R, Dieterich M, et al. Endothelin-A receptor blockade prevents left ventricular hypertrophy and dysfunction in salt-sensitive experimental hypertension. Circulation. 2002;106:2305-2308.

6. Zolk O, Quattek J, Sitzler G, et al. Expression of endothelin-1, endothelin-converting enzyme, and endothelin receptors in chronic heart failure. Circulation. 1999;99:2118-2123.

7. Anand I, McMurray J, Cohn JN, et al. Long-term effects of darusentan on leftventricular remodelling and clinical outcomes in the EndothelinA Receptor Antagonist Trial in Heart Failure (EARTH): randomised, double-blind, placebocontrolled trial. Lancet. 2004;364:347-354.
8. Mathews WB, Murugesan N, Xia J, et al. Synthesis and in vivo evaluation of novel PET radioligands for imaging the endothelin-A receptor. J Nucl Med. 2008;49:1529-1536.

9. Guide for the Care and Use of Laboratory Animals. Washington, DC: Government Printing Office; 1985. NIH publication 86-23.

10. Higuchi T, Nekolla SG, Jankaukas A, et al. Characterization of normal and infarcted rat myocardium using a combination of small-animal PET and clinical MRI. J Nucl Med. 2007;48:288-294.

11. Taki J, Higuchi T, Kawashima A, et al. Detection of cardiomyocyte death in a rat model of ischemia and reperfusion using ${ }^{99 \mathrm{~m} T c-l a b e l e d ~ a n n e x i n ~ V . ~ J ~ N u c l ~ M e d . ~}$ 2004;45:1536-1541.

12. Higuchi T, Taki J, Nakajima K, Kinuya S, Namura M, Tonami N. Time course of discordant BMIPP and thallium uptake after ischemia and reperfusion in a rat model. J Nucl Med. 2005;46:172-175.

13. Higuchi T, Bengel FM, Seidl S, et al. Assessment of alphavbeta3 integrin expression after myocardial infarction by positron emission tomography. Cardiovasc Res. 2008;78:395-403.

14. McMurray JJ, Ray SG, Abdullah I, Dargie HJ, Morton JJ. Plasma endothelin in chronic heart failure. Circulation. 1992;85:1374-1379.

15. Shimoyama H, Sabbah HN, Borzak S, et al. Short-term hemodynamic effects of endothelin receptor blockade in dogs with chronic heart failure. Circulation. 1996;94:779-784.

16. Sakai S, Miyauchi T, Yamaguchi I. Long-term endothelin receptor antagonist administration improves alterations in expression of various cardiac genes in failing myocardium of rats with heart failure. Circulation. 2000;101:2849-2853.

17. Miyauchi T, Sato R, Sakai S, et al. Endothelin-1 and right-sided heart failure in rats: effects of an endothelin receptor antagonist on the failing right ventricle. J Cardiovasc Pharmacol. 2000;36(suppl 1):S327-S330.

18. Mulder P, Boujedaini H, Richard V, et al. Long-term survival and hemodynamics after endothelin-a receptor antagonism and angiotensin-converting enzyme inhibition in rats with chronic heart failure: monotherapy versus combination therapy. Circulation. 2002;106:1159-1164.

19. Mylona P, Cleland JG. Update of REACH-1 and MERIT-HF clinical trials in heart failure. Cardio.net Editorial Team. Eur J Heart Fail. 1999;1:197-200.

20. Kalra PR, Moon JC, Coats AJ. Do results of the ENABLE (Endothelin antagonist bosentan for lowering cardiac events in heart failure) study spell the end for nonselective endothelin antagonism in heart failure? Int J Cardiol. 2002;85:195-197.

21. Fraccarollo D, Galuppo P, Bauersachs J, Ertl G. Collagen accumulation after myocardial infarction: effects of ETA receptor blockade and implications for early remodeling. Cardiovasc Res. 2002;54:559-567.

22. Mencarelli M, Pecorelli A, Carbotti P, Valacchi G, Grasso G, Muscettola M. Endothelin receptor A expression in human inflammatory cells. Regul Pept. 2009; 158:1-5.

23. Katwa LC. Cardiac myofibroblasts isolated from the site of myocardial infarction express endothelin de novo. Am J Physiol Heart Circ Physiol. 2003;285:H1132H1139.

24. Vignon-Zellweger N, Heiden S, Miyauchi T, Emoto N. Endothelin and endothelin receptors in the renal and cardiovascular systems. Life Sci. 2012;91:490-500.

25. Kedzierski RM, Grayburn PA, Kisanuki YY, et al. Cardiomyocyte-specific endothelin A receptor knockout mice have normal cardiac function and an unaltered hypertrophic response to angiotensin II and isoproterenol. Mol Cell Biol. 2003;23:8226-8232.

26. Zhang Y, Li L, Hua Y, et al. Cardiac-specific knockout of ET(A) receptor mitigates low ambient temperature-induced cardiac hypertrophy and contractile dysfunction. J Mol Cell Biol. 2012;4:97-107.

27. Aleksic S, Szabo Z, Scheffel U, et al. In vivo labeling of endothelin receptors with [C-11]L-753,037: studies in mice and a dog. J Nucl Med. 2001;42:12741280.

28. Johnström P, Fryer TD, Richards HK, et al. Positron emission tomography using F-18-labelled endothelin-1 reveals prevention of binding to cardiac receptors owing to tissue-specific clearance by ETB receptors in vivo. $\mathrm{Br} J$ Pharmacol. 2005; 144:115-122.

29. Johnström P, Rudd JHF, Richards HK, et al. Imaging endothelin ET(B) receptors using [F-18]-BQ3020: In vitro characterization and positron emission tomography (MicroPET). Exp Biol Med. 2006;231:736-740. 